\title{
High-depth resequencing reveals hybrid population and insecticide resistance characteristics of fall armyworm (Spodoptera frugiperda) invading China
}

Lei Zhang ${ }^{1 \#}$, Bo Liu ${ }^{1 \#}$, Weigang Zheng ${ }^{1 \#}$, Conghui Liu ${ }^{1 \#}$, Dandan Zhang ${ }^{2 \#}$, Shengyuan Zhao ${ }^{2 \#}$, Pengjun $\mathrm{Xu}^{3,4}$, Kenneth Wilson ${ }^{4,1}$, Amy Withers ${ }^{4}$, Christopher M. Jones ${ }^{5}$, Judith A. Smith ${ }^{6}$, Gilson Chipabika $^{7}$, Donald L. Kachigamba ${ }^{8}$, Kiwoong Nam ${ }^{9}$, Emmanuelle d'Alençon ${ }^{9}$, Bei Liu ${ }^{1}$, Xinyue Liang ${ }^{1}$, Minghui $\mathrm{Jin}^{1}$, Chao $\mathrm{Wu}^{1}$, Swapan Chakrabarty ${ }^{1}$, Xianming Yang ${ }^{2}$, Yuying Jiang ${ }^{10}$, Jie Liu ${ }^{10}$, Xiaolin Liu ${ }^{11}$, Weipeng Quan ${ }^{12}$, Guirong Wang ${ }^{1}$, Wei Fan ${ }^{1 *}$, Wanqiang Qian ${ }^{1 *}$, Kongming $\mathrm{Wu}^{2 *}$, Yutao Xiao ${ }^{1 *}$

1 Lingnan Guangdong Laboratory of Modern Agriculture, Genome Analysis Laboratory of the Ministry of Agriculture, Agricultural Genomics Institute at Shenzhen, Chinese Academy of Agricultural Sciences, Shenzhen 518120, China

2 State Key Laboratory for Biology of Plant Diseases and Insect Pests, Institute of Plant Protection, Chinese Academy of Agricultural Sciences, Beijing 100193, China

3 Tobacco Research Institute of Chinese Academy of Agricultural Sciences, Qingdao 266101, China

4 Lancaster Environment Centre, Lancaster University, Lancaster LA1 4YQ, UK

5 Malawi-Liverpool-Wellcome Trust Clinical Research Programme, Blantyre, Malawi

6 School of Forensic and Applied Sciences, University of Central Lancashire, Preston PR1 2HE, UK

7 Zambia Agriculture Research Institute (ZARI), Lusaka, Zambia

8 Department of Agricultural Research Services (DARS), Bvumbwe Research Station, P. O. Box 5748, Limbe, Malawi

9 DGIMI, INRA, Univ. Montpellier, 34095, France

10 National Agro-Tech Extension and Service Center, Beijing 100125, China

11 Novogene Bioinformatics Institute, Beijing 100083, China

12 Gandomics Biosciences, Co., Ltd, Beijing 102206, China

"These authors contributed equally to this work.

*Correspondence: Yutao Xiao (xiaoyutao@caas.cn), Kongming Wu (wukongming@caas.cn), Wanqiang Qian (qianwanqiang@caas.cn), Wei Fan (fanwei@caas.cn). 


\begin{abstract}
The rapid wide-scale spread of fall armyworm (Spodoptera frugiperda) has caused serious crop losses globally. However, differences in the genetic background of subpopulations and the mechanisms of rapid adaptation behind the invasion are still not well understood. Here we report a 393.25-M chromosome-level genome assembly of fall armyworm with scaffold N50 of $13.3 \mathrm{M}$ consisting of 23281 annotated protein-coding genes. Genome-wide resequencing of 105 samples from 16 provinces in China revealed that the fall armyworm population comprises a complex inter-strain hybrid, mainly with the corn-strain genetic background and less of the rice-strain genetic background, which highlights the inaccuracy of strain identification using mitochondrial or Tpi genes. An analysis of genes related to pesticide- and Bt-resistance showed that the risk of fall armyworm developing resistance to conventional pesticides is very high, while remaining currently susceptible to Bt toxins. Laboratory bioassay results showed that insects invading China carry resistance to organophosphate and pyrethroid pesticides, but are sensitive to genetically modified maize expressing Cry1 Ab in field experiments. Additionally, we found that two mitochondrial fragments are inserted into the nuclear genome, and the insertion event occurred after the differentiation of the two strains. This study represents a valuable advancement toward the analysis of genetic differences among subpopulations and improving management strategies for fall armyworm.
\end{abstract}

\title{
Keywords
}

Spodoptera frugiperda; subpopulations; population structure; resistance risk; gene insertion

\section{Introduction}

The fall armyworm, Spodoptera frugiperda (J.E. Smith), is a polyphagous pest that is native to tropical and subtropical America, with a strong capacity for migration and reproduction ${ }^{1-4}$. The pest was first detected in Africa in $2016^{5}$ and spread to 44 African countries within two years. It was first detected in Asia in July 2018, and so far it has spread to 13 countries in the region (https://gd.eppo.int/taxon/LAPHFR/distribution). Such rapid spread poses a global threat to food production. The strong environmental adaptability of fall armyworm is not only reflected in its polyphagy for a wide range of host plants ${ }^{6}$, but also in its evolution of resistance to chemical pesticides and genetically modified crops expressing Bacillus thuringiensis $(\mathrm{Bt})$ toxins $^{7-16}$. Studies have shown that the genes related to detoxification and metabolic processes in the fall armyworm have exhibited obvious expansion ${ }^{17-18}$. In addition, there are two morphologically identical, but 
genetically distinct, subpopulations or strains of fall armyworm, the rice-strain (R-strain) and the corn-strain (C-strain), which differ in their host plant selection and sex pheromone composition $^{19-22}$. However, there is no absolute mating barrier between the two strains and productive hybridization has been confirmed in both laboratory and field studies ${ }^{23-24}$.

At present, a number of field-evolved resistant populations of fall armyworm have been detected, including resistance to a variety of chemical pesticides and Bt crops, and the level of resistance is increasing ${ }^{25-29}$. The mechanisms of resistance to pesticides are mainly due to variation in receptor genes, such as amino acid mutations in the ryanodine receptor (RyR) (diamide), acetylcholinesterase (AChE) (organophosphate), voltagegated sodium channel (VGSC) (pyrethroids), and so on ${ }^{30-32}$. In addition, the frame-shift mutation resulting in early termination of the $A B C C 2$ gene, caused by a 2-bp insertion, is linked to resistance to Cry $1 \mathrm{Fa}^{33}$. Field-evolved strains resistant to Vip3Aa20 were obtained by screening homozygous resistance loci in $\mathrm{F}_{2}$ generations in the laboratory ${ }^{34}$. The resistance mechanisms of other Cry toxins from Bt are still unknown. Clarifying the development of pesticide- and Bt-resistance in fall armyworm would be helpful in providing scientific support for the commercialization of genetically modified crops and Bt biopesticides.

Recent studies have indicated that the molecular identification of the two strains of fall armyworm is dependent on which markers are used ${ }^{35-37}$. The early molecular markers based on mitochondrial Cytochrome Oxidase Subunit I (COI) and Z-chromosome-linked Triosephosphate isomerase (Tpi) genes failed to accurately assign strain identification ${ }^{38-41}$. The dominant population of fall armyworm invading Africa and Asia were speculated to be hybrid populations of the female $\mathrm{R}$-strain and male C-strain, based on these two molecular markers ${ }^{42}$. In addition, an Africa-specific haplotype, different from those of native Americas, was also reported in African and Chinese samples based on the Tpi gene ${ }^{18,40}$, which makes strain identification and population genetic structure more complicated. Therefore, a genome-wide analysis of the genetic characteristics of invasive fall armyworm is becoming imperative. Although several versions of the fall armyworm genome have now been published ${ }^{17-18,43-44}$, the different mechanisms directly related to the two strains are unclear, and the debate about strain identification requires further genomic support and explanation. Here we report a chromosome-level genome sequence of a male moth from an inbred fall armyworm strain, representing a C-strain $C O I$ and an Africa-specific Tpi haplotype which was different from the Western Hemisphere (henceforth American) R-strain and C-strain. We also re-sequenced 105 fall armyworm samples from 16 Provinces in China, as well as four samples collected from two African countries (Zambia and Malawi). The genome-wide genetic backgrounds of the invading fall armyworm samples were compared, and the insecticide-resistance risk was assessed based on analysis of potential resistance-related genes. Comparative genomic analyses of these data help to reveal the resistance-related mechanisms and the population genetic characteristics of fall armyworm, which may facilitate its future management. 


\section{Results}

\section{High-quality genome landscape of fall armyworm.}

Genomic DNA of fall armyworm was extracted from a male moth of an inbred laboratory-reared strain, and sequencing reads were obtained using both PacBio and Illumina technologies and were assembled after filtering out the low-quality and duplicated reads. A total of $24.72 \mathrm{G}$ PacBio long reads and $162.4 \mathrm{G}$ of high-quality Illumina reads were generated, representing an $\sim 460 \times$ coverage of the fall armyworm genome. Through wtdbg2 $2^{45}$, the final genome was assembled into 777 contigs with size of $393.25 \mathrm{M}$ and contig N50 length of $5.6 \mathrm{M}$ (longest, $18 \mathrm{M}$ ), including a complete mitochondrial sequence. The assembled genome size was close to the estimated size of $395 \mathrm{M}$ based on k-mer depth distribution analysis, which was also similar to that of flow cytometry $(396 \pm 3 \mathrm{M})^{17}$. After interaction analysis based on a total of $78 \mathrm{G}$ data obtained through Hi-C sequencing, 497 contigs were concatenated to 31 chromosomes with the scaffold N50 of 13.3 M, accounted for $98.67 \%$ of total genome length (Supplementary Fig. 1, Supplementary Table 1). By aligning the Illumina data with the assembled fall armyworm genome, the mapping rate and coverage were $98.76 \%$ and $99.68 \%$ respectively, which showed the accuracy and high integrity of genome assembly. The genome size reported in this study is intermediate between those of previously published fall armyworm versions, but the genome is nearly $140 \mathrm{M}$ smaller than that recently published by Liu et al. (2019). By genome collinearity analysis, the alignment showed that more than $98 \%$ of the current assembled genome was consistent with previously published versions (Supplementary Table 2), indicating that the genome integrity of this study was high, and the previous assembled genome with larger size was mainly caused by high heterozygosity of sequenced samples.

By combining homology-based and de novo approaches, we identified $\sim 27.18 \%$ of repetitive elements in the assembled fall armyworm genome. Among the known repeat families, LINE constituted the most abundant repeat family, representing $8.66 \%$ of the repetitive sequences, while LTR was only $1.38 \%$ (Supplementary Table 3). To annotate the fall armyworm genome, we performed deep transcriptome sequencing of larvae, pupae, male and female moths, including three different developmental stages, which generated $98.4 \mathrm{G}$ of RNA sequencing data. By combing homologue-based, ab initio and transcriptome-based approaches, we predicted 23281 protein-coding genes (gene models) in the fall armyworm genome, which is greater than the number of predicted genes in other lepidopteran genomes that have so far been published ${ }^{46-51}$. The average CDS length is $1476 \mathrm{bp}$, with 5.39 exons per gene and an average intron size of $1165 \mathrm{bp}$, which is larger than those in previously published fall armyworm genomes. More than $85.5 \%$ of the predicted coding sequences were supported by transcriptome sequencing data. Further assessment of assembly integrity based on Benchmarking Universal Single-Copy Ortholog (BUSCO) analysis shows that the current genome covered 98.2\% complete BUSCO genes (Supplementary Table 4), indicating the high accuracy of the gene predictions. 
Comparative analysis of orthogroups of nine Lepidoptera species and Drosophila melanogaster (Diptera) were performed. Among them, 17571 groups were found in the current genome of fall armyworm, mainly in the number of other and unassigned genes. Compared with Spodoptera litura, S. frugiperda has more species-specific genes, and the number of unassigned genes is much greater than that of S. litura (Fig. 1a). Phylogenomic analyses of the ten species were conducted using 1571 single-copy genes. As shown in Figure 1a, the phylogenetic tree reflects the taxonomic relationship and phylogenetic status of the different species. Three species of Noctuidae, including S. frugiperda, formed one group, which then clustered with Bombyx mori (Bombycidae) and Manduca sexta (Sphingidae). Two butterflies Danaus plexippus and Heliconius melpomene (both Nymphalidae) clustered together as an outer branch, while Plutella xylostella (Plutellidae) is the outermost branch of Lepidoptera (Fig. 1a).

\section{Insertion of mitochondrial fragments into nuclear genome in a recent evolution event.}

We found that two mitochondrial fragments, with sequence lengths of $1.5 \mathrm{~K}$ (partial COI gene and $N A D H 2$ gene) and $1.6 \mathrm{~K}$ (partial $N A D H 2$ gene and $12 \mathrm{~S}$ rRNA gene), were inserted into the nuclear genome, separated by a $4 \mathrm{~K}$ segment of the nuclear genome (Fig. 1b). The total length of a $\sim 7.3 \mathrm{~K}$ fragment, including two inserted fragments, was supported by more than 28 original reads of PacBio data. The lengths of all 28 original reads were longer than $20 \mathrm{~K}$ and completely covered the $7.3 \mathrm{~K}$ fragment. However, the two insertions were not found in other published fall armyworm genomes. In order to verify the accuracy of this result, we designed four primers based on flanking sequences of four connection points, and the results of PCR amplification confirmed the existence of the insertion. In addition, four primers were applied to detect the insertion in 173 fall armyworm samples collected from different regions of China and it was found that the insertion was only present in $26.01 \%$ of all samples (Supplementary Table 5). At the same time, the resequencing Illumina data of 109 fall armyworm samples in this study also showed that there were varying numbers of reads covering the four junction points in 30 samples, and the percentage of samples with inserted reads was $28.5 \%$ (Supplementary Table 6). Both the PCR and resequencing results showed that the insertion was not present in all samples, suggesting that it was a late evolution event.

Moreover, the genotype of the two inserted mitochondrial fragments were identical with those of the $\mathrm{C}$-strain, indicating that the insertion occurred after the differentiation of the R-and C-strains, and was more likely to be a random recombination event between the two strains. Further analysis indicated that two mitochondrial fragments were inserted into the intron region of lysine-specific demethylase $3 \mathrm{~B}$ (Kdm3B) gene, which is not likely to affect the expression of the gene. The inserted partial $\mathrm{COI}$ and $\mathrm{NADH} 2$ gene fragments were also not tend to express and thus play a mitochondrial related function. However, such two fragments could be used to develop markers to identify specific populations and used for further evolutionary events of fall armyworm. 


\section{Hybrid genetic background of fall armyworm population invading China.}

In order to identify the specific genetic background of the invading fall armyworm samples in China, 105 samples from 16 provinces were re-sequenced, as well as four samples from two countries in Africa (Zambia and Malawi). A total of $\sim 10 \mathrm{G}$ genomic Illumina data were generated for each sample, with a total coverage of about $25 \times$ genome size. Firstly, we analyzed the whole mitochondrial genome sequences of all samples. A total of 208 different SNP loci were screened based on the published mitochondrial sequences of both the American R-strain (AXE) and C-strain (ASW). The original Illumina reads of each sample were mapped to the reference mitochondrial genomes, and the genotype of re-sequenced samples were identified according to these SNP loci. We found that most of the samples were assigned to R-strain, and all four samples from Africa were C-strain, while only four out of 105 samples in China were assigned to C-strain based on the mitochondrial genome (Fig. 2a). The results were similar to those of the 173 Chinese fall armyworm samples identified by PCR based on the COI gene in this study. The proportion of C-strain was only about $10 \%$ (Supplementary Table 5), which is consistent with previous reports ${ }^{42}$.

Next, we analyzed the Tpi gene, which is commonly used in strain identification ${ }^{36-37}$. By comparing the full length Tpi gene of published R-strain and C-strain fall armyworm, 22 SNP loci were found. The genotypes of all samples were analyzed by calculating the ratio of SNPs compared to the American reference sequences (AXE and ASW). The results showed that most fall armyworm samples collected from China contained more C-strain SNP loci, as did the Malawi samples (AFR14, AFR15), but not those from Zambia (AFR4, AFR5) which contained approximately $50 \%$ of R-strain SNP loci. Genotypes of seven samples were identical to the American C-strain (ASW) reference (Fig. 2b), possibly due to them being females or homozygous genotypes. The remainder of the samples contained a small proportion of R-strain genotypes or heterozygous SNPs. However, none of the samples were found to be identical to the American R-strain genotype (AXE). We designed a pair of primers to amplify a Tpi fragment that included Tpi-exon3, exon4 and intron3. We used the primers to screen 173 samples and to analyze 10 different SNP loci of each sample by comparing to R-strain and C-strain genotypes reported previously (Fig. 3, Supplementary Table 5). The results showed that almost all of the samples were C-strain genotypes except three samples (G-GXW11, G-GXW13, G-EP6) were identified as Africa-specific haplotype, in which 6 of 10 SNPs were identical to R-strain, while 4 were identical to C-strain, and 10 unique SNPs were significantly different from known R- or C-strain genotypes (Fig. 3). It can also be seen from the Figure $2 b$ that the genotyping results of AFR4-5 based on full-length Tpi gene, which represents the Africa-specific strain, are quite different from the rest of the samples in containing $>40 \%$ R-strain SNPs. In summary, our genotyping results show that there are obvious contradictions between strain identification using mitochondrial and nuclear $T p i$ gene markers.

In order to clarify the genetic background of fall armyworm populations invading China, we 
mapped the published Illumina data of R-strain (AXE) and C-strain (ASW) to the reference genome of this study. Both AXE and ASW strains were from colonies that have been reared in laboratory for over 10 years, which could be considered to represent R-strain and C-strain populations, and finally a total of 707353 homozygous SNPs between the two strains were screened. The genotypes of 109 re-sequenced samples were analyzed by comparing Illumina data of each sample with these SNPs (Fig. 2c). The results showed that all the samples, including the four from Africa, had more than $70 \%$ of the genetic background of the American C-strain, but there were no individuals completely identical with C-strain (ASW) genotype. The proportion of R-strain SNPs was less than $15 \%$, and the remaining $15 \%$ were heterozygous. The results showed that fall armyworm invading China have a dominant percentage of the C-strain background at the whole genome level. By comparing the results of the mitochondrial genome, Tpi gene and genome-wide identification, it becomes apparent that there is no obvious correlation between the mitochondrial and whole genome genotype. Tpi genotyping shows results more similar to those of the whole genome. However, there is a high proportion of heterozygosity at strain-specific loci, perhaps due to male individuals with ZZ chromosomes, which reduces the accuracy of genotyping based on the Tpi gene. Moreover, the presence of Africa-specific Tpi-haplotype increases the complexity of using this marker for identification. Strain analysis based on the whole genome reflects a more accurate approach.

In addition, 105 re-sequenced Chinese samples were collected from different regions of 50 cities distributed across 16 provinces. The collection time and sites coincided almost perfectly with the spreading invasion of fall armyworm in China. However, there was no obvious correlation between the time or site of collection and the genetic structure of the fall armyworm population (Fig. 2). Almost all samples have similar genomic backgrounds, which suggests that the invading population may originate from a single genetic source and there is no evidence for genomic selection during the invasion. However, the analysis based on the mitochondrial genome (Fig. 2a) and the Tpi gene (Fig. 2b) might erroneously suggest a more heterogeneous population genetic structure.

\section{Fall armyworm is developing high risk of resistance to conventional pesticides.}

Insecticide resistance evolution is one of the most challenging problems to be solved in the control of fall armyworm. Identifying resistance-related genes is helpful for the monitoring and prevention of fall armyworm outbreaks. We selected 14 previously reported resistance-related genes of lepidopteran pests (Supplementary Table 7) and scanned the re-sequenced samples to analyze variation in target genes. The results showed that all the target genes had multiple variation sites with high frequency of SNPs in the coding sequence (CDS) region (Fig. 4a). Sequence diversity of CYP450 and AChE were both reached 0.01, while the rest genes were no more than 0.005 (including heterozygotes), suggesting a great potential risk of resistance evolution. 
Studies have shown that the amino acid substitutions in AChE (A201S, G227A, F290V), VGSC (T929I, L932F, L1014F) and RyR (I4790M, G4946E) result in resistance to organophosphate, pyrethroid and diamide insecticides, respectively. The results of variation scanning of the re-sequenced samples showed that resistance mutations were found at the first (AA201) and third locus (AA290) of AChE gene. Among them, the first locus had 17.1\% heterozygous mutations, and the third locus had 29.7\% homozygous resistance mutations and 58.2\% heterozygous mutations. And no resistant mutations were detected at corresponding sites of VGSC and RyR gene in any samples (Table 1). We designed primers to detect the resistant mutation sites in AChE in 173 Chinese samples by PCR amplification and Sanger sequencing. The results were similar to the Illumina data, showing approximately $25 \%$ homozygous and $50 \%$ heterozygous variation at the third locus (AA290).

We determined the $\mathrm{LC}_{50}$ s to 14 insecticides for two Chinese fall armyworm populations collected from Yunnan Province (Supplementary Table 8). The results showed that the $\mathrm{LC}_{50} \mathrm{~s}$ for both fall armyworm populations to fenvalerate, malathion, chlorpyrifos were relatively high. The $\mathrm{LC}_{50} \mathrm{~S}$ of chlorantraniliprole and cyantraniliprole were low along with emamectin benzoate and E-MBI (Fig. 5). The resistance levels of the two populations to pyrethroids and organophosphate pesticides were very high; in particular, the resistance ratio to chlorpyrifos of both populations were more than 300-fold compared to a laboratory susceptible population that was sampled in $1975^{52}$ (Fig. 4b, Supplementary Table 9). These results provide a susceptible baseline for fall armyworm populations invading China to different pesticides, which can provide guidance for resistance monitoring and pesticide management strategies.

\section{Fall armyworm invading China are currently sensitive to Bt toxin in field-evolved experiment.}

The insertion of $2 \mathrm{bp}$ in the $A B C C 2$ of fall armyworm was reported to cause a frame-shift mutation and results in resistance to $\mathrm{Cry} 1 \mathrm{Fa}^{33}$. We did not detect the same insertion mutation in 105 re-sequenced samples nor in 173 samples screened by using PCR and Sanger sequencing. Although the percentage of SNPs in the CDS region of other Bt receptors such as $S R$ - $C$ (scavenger receptor class $\mathrm{C}$ gene, a specific receptor for Vip3Aa in Sf9 cells), TSPAN1 and other ABC gene-family related to Cry toxin were also very high (Fig. 4a), no reported resistant mutation were found in any target resistance genes.

The field tests showed that fall armyworm samples invading China were sensitive to GM maize expressing Cry1 Ab compared with control group, which is accordant with the result of resistant genes scanning. The damage assessment on larval density, percentage of damaged plants and average damage ratings of GM maize were significantly lower than those of the control group (Fig. $4 \mathrm{c})$, which indicated that the GM maize expressing Cry1 Ab currently has good control effects on the invading population of fall armyworm in China. 


\section{Discussion}

The rapid spread of the fall armyworm has attracted popular attention worldwide. Accurate identification of its genetic characteristics (strain and pesticide resistance properties) has a direct and practical importance in terms of risk assessment and control strategies. A genome-wide analysis can reveal more in-depth genetic information than conventional gene-level analyses. The results of this study show that the fall armyworm invading China had a genetic background with dominant American corn-strain genotype and might be descendants of an inter-strain hybrid population. Most of the fall armyworm samples invading China were detected and collected from corn and sugarcane, which are more likely to show the characteristics of C-strain host plants. Along the invasion path of the migratory fall armyworm, there are large-scale rice planting areas in Southeast Asia and central China, however, so far there are few reports of serious damage to rice caused by fall armyworm (http://www.fao.org/fall-armyworm). The traditional R-strain fall armyworm in the Americas mainly feeds on turf grass, and there are also some reports of damage to rice. In addition, the traditional R-strain Tpi genotype has not been detected in any of the samples collected from Africa and Asia, so we speculate that the American R-strain fall armyworm did not invade Africa or Asia, including China.

Mitochondrial $\mathrm{COI}$ and $T p i$ genes are commonly used as markers for the identification of fall armyworm strain. Current studies have shown that the mitochondrial genome is clearly divided into two strains. Because of their hybridization, identification based on the maternally-inherited mitochondrial genotype is inaccurate and the insertion of two C-strain mitochondrial fragments in this study further confirms this inaccuracy. Therefore, it is not possible to accurately infer fall armyworm strain status based on the complete mitochondrial genome or the single COI mitochondrial gene, as suggested previously ${ }^{40-41}$. The Tpi gene, located in the nuclear genome, is more suitable for strain identification and 10 SNPs in this gene can distinguish between R- or C-strains fall armyworm in the Americas ${ }^{37}$. In this study, we found that the AT/GC SNP located at Tpi-intron3 (P173/174) was not linked with the other eight SNPs and was not specific to either the $\mathrm{R}$ - or C-strain genotypes precluding these two SNPs as diagnostic markers. In addition, the TT/CC SNP located at Tpi-exon4 (P379/385) was associated with sequence variation in Tpi-intron4 (Fig. 3, Supplementary Fig. 2). A special (Africa-specific) haplotype of the Tpi gene was identified in samples collected from southern-east Africa, which was also detected in some Chinese samples. For this specific haplotype, it is not identical to either American R- or C-strain genotypes, although it was tentatively designated as R-strain based on E4 ${ }^{183}$ site (equal to P370 in Fig. 3 in this study) in previous studies ${ }^{37}$. Our genome-wide SNP analysis revealed that this haplotype contained more C-strain SNPs than R-strain.

This analysis shows that the fall armyworm shows a complicated population genetic structure. For example, the sample used for the genome sequencing in this study represents a combination of the special Tpi haplotype and C-strain COI. We also found combinations of the R-strain COI and 
special Tpi (sample G-XW13) as well as heterozygous forms of the Tpi-special and Tpi-C with the R-strain COI in two samples (G-GXW11, G-EP6). These combinations of different genotypes and extensive gene exchange show that the genetic boundaries between two traditional (American) Rand $\mathrm{C}$-strains are obscure. The insertion of two $\mathrm{C}$-strain mitochondrial fragments might be caused by random hybridization between different genotypes.

The rapid evolution of insecticide resistance and the increasing levels of resistance observed in fall armyworm populations needs attention. In this study, mutations related to organophosphate pesticide resistance were detected in AChE gene. Although some mutation sites were detected as heterozygous in most samples at present, the frequency of resistant mutation sites will increase greatly under the selection pressure caused by application of related pesticides in field. The pesticide bioassay results showed that armyworms invading China have evolved high levels of resistance to chlorpyrifos, in particular, which was consistent with the results of molecular scanning of insecticide resistance-related genes. However, the fall armyworms invading China are currently sensitive to GM maize expressing Cry1 $\mathrm{Ab}$ in field experiments, and are also sensitive to other Bt toxins in the laboratory, according to previous studies ${ }^{53}$. At present, GM maize shows better application prospects in controlling fall armyworm in China, larval density and damage rate of GM maize were significantly less than that of normal plants, though this crop is currently not registered for use in the country.

This study provides a high-quality reference genome that demonstrates a genomic feature different from the traditional (American) C- or R-strain genotypes, as well as more comprehensive gene annotation. According to our results, commonly used strain identification of fall armyworms by mitochondrial or Tpi markers is limited or even inaccurate. We present resequencing data for 105 fall armyworm individuals invading China. The samples cover different regions and times during 2019, providing basic materials for global population genetic analyses. Baseline resistance data for Chinese fall armyworm populations is provided to 14 common pesticides, providing guidance for the control and resistance monitoring of fall armyworm. Small-scale field experiments in this study suggest that that fall armyworm are currently susceptible to GM maize, and these results could provide an important application reference for commercial planting of Bt maize in China. There are other important issues that remain for further exploitation using this whole genome approach, such as identifying the genes involved in polyphagy, migratory capability and olfaction, which could provide valuable tools for the future management of fall armyworms.

\section{Methods}

Genome sequencing and assembly. The fall armyworm samples were collected from maize fields in Lusaka, Zambia, in 2017 and reared to produce an inbred strain. One male moth, derived from seven successive generations of single-pair sib mating, was selected for extracting genomic 
DNA and constructing Illumina 350-bp and PacBio 20-kb insert libraries. Sequencing was performed on Illumina HiSeq 2500 and PacBio SMRT platforms, respectively. Two 3rd instar larvae were selected for Hi-C library construction and then sequenced on a HiSeq 2500 platform (Illumina). In addition, three 5th instar larvae, three pupae, three female moths and three male moths were used for RNA sequencing on an Illumina HiSeq 2500 sequencer. All the above samples were from the same inbred strain.

The raw PacBio reads longer than $5 \mathrm{~Kb}$ were assembled into contigs using software wtdbg $2^{45}$. Arow v2.1.0 software was used to correct assembly errors after comparing contigs with PacBio reads by pbalign v0.4.1. The Illumina raw reads were filtered by trimming the adapter and low-quality regions, resulting in high-quality reads. Then clean Illumina reads were aligned to the assembled contigs by BWA v0.7.17, and single base errors in the contigs were corrected by Pilon v1.21.

After removing adapter and low-quality sequences, Hi-C sequencing data were aligned to assembled contigs with Hic-Pro, then the unmapped paired, dangling paired, self-circle and dumped reads were filtered out (https://github.com/nservant/HiC-Pro). According to the restriction enzyme (DpnII) sites on contigs, we clustered the linkage group of contigs based on agglomerative hierarchical clustering method by using LACHESIS $^{54}$ (https://github.com/shendurelab/LACHESIS), and then these contigs were clustered into chromosomes.

Gene annotation and prediction. A de novo repeat library of fall armyworm was constructed by RepeatModeler v. 1.0.4. TEs were identified by RepeatMasker v4.0.6 using both de novo library and Repbase library, and Tandem repeats were predicted using Tandem Repeats Finder ${ }^{55}$ v4.07b. The gene models were predicted by EVidence Modeler $^{56}$ v1.1.1, combined with ab initio predictions, homology-based searches, and RNA sequencing alignments. Predicted gene models supported by at least one of the annotations using UniProt datbase, NR database, and RNA-seq data were retained. Then gene functional annotation was performed by aligning the protein sequences to the NCBI NR, UniProt, eggNOG, and KEGG databases with BLASTp v2.3.0+.

Phylogenetic tree construction and genomic comparison. Orthologous and paralogous groups of 10 species (Drosophila melanogaster, Plutella xylostella, Bombyx mori, Manduca sexta, Danaus plexippus, Heliconius melpomene, Operophtera brumata, Helicoverpa armigera, Spodoptera frugiperda, Spodoptera litura) with published genomes were analyzed by OrthoFinder v2.3.1 with default parameters. Orthologous groups that contain single-copy genes for each species were selected to construct the phylogenetic tree. The multi-sequence alignment of proteins was accomplished by MUSCLE ${ }^{57}$ v3.8.31. The Neighbor-Joining (NJ) phylogenetic tree was constructed by MEGA v7.0.14. The current assembled genome was aligned with two published versions of fall armyworm genomes by nucmer software with cutoff of identity $>80 \%$ and coverage $>80 \%$. Unique alignment comparison was conducted with the parameters "delta-filter -i 
85 -1 10 -r $-\mathrm{q}$ ", while multiple alignment comparison were conducted with the parameters "delta-filter -i 85 -1 10".

Sampling for resequencing and population genetic study. A total of 105 Chinese fall armyworm samples were used for resequencing, including four samples of two inbred strains (cdcc and cdyc) collected from Yunnan Province and reared for multiple generations in the laboratory. All samples were collected as larvae on maize or sugarcane from 50 cities of 16 provinces (autonomous regions or municipalities) of China. The larvae were fed with fresh maize leaves and brought back to the laboratory under ambient conditions during transportation. Larval bodies were cleaned and then stored in a freezer at $-80{ }^{\circ} \mathrm{C}$. The detailed sample information is shown in Supplementary Table 10 and the sample distribution in China is shown in Supplementary Figure 3. In addition, four fall armyworm samples from Africa were also used for resequencing, including two samples (AFR4-5) from the same inbred strain (AFR2017) as the genome sequencing in this study, and another two samples (AFR14-15) which were collected from maize fields in Bvumbwe, Malawi, in January 2019, which is also an inbred strain (AFR2019) reared in laboratory. After DNA extraction of each sample, a 350-bp insert library were constructed and paired-end sequencing was performed following the standard Illumina protocol. Sequence reads from microorganisms and host plants in raw data were removed before analysis.

In addition to re-sequenced samples, another 173 fall armyworm samples from 21 provinces in China were used for strain identification and molecular detection based on PCR amplification and Sanger sequencing. The samples were collected from the field as larvae or adult moths. The detailed sample information is shown in Supplementary Table 5 and the sample distribution in China is shown in Supplementary Figure 3. Mitochondrial COI and Tpi markers were used for strain identification. $\mathrm{ABCC} 2, \mathrm{AChE}$ genes were detected based on primers designed according to published mutation sites $^{31-33}$. Mitochondrial insertion fragment detection was conducted using primers designed in this study. All primer sequence information in this study is shown in Supplementary Table 11.

Read mapping and SNP calling. The Illumina raw reads from re-sequenced samples were filtered using clean_adapter and clean_lowqual software (https://github.com/fanagislab/common_use), resulting in high-quality reads with an average error rate of $<0.01$. Then, the high quality reads were aligned to the fall armyworm reference genome and mitochondrial genome sequences by the Burrows-Wheeler Transform Alignment (BWA) software $^{58}$ package v0.7.5a with default parameters. Alignments for each sample were processed by removing duplicate reads using samtools ${ }^{59}$ software package v1.3. The mpileup function in samtools was used to generate mpileup files for each sample. Bcftools-vcftools ${ }^{60}$ was used to identify SNPs and small Indels. Several criteria were considered in SNP filtering: (1) a read mapping score higher than 40; (2) minimum coverage greater than 10.

Bioassays of insecticides and Bt maize in the field. Bioassays were conducted by a topical 
application procedure ${ }^{61}$. Two inbred strains of Chinese fall armyworm populations (cdcc, cdyc) were tested using 14 types of pesticide commonly used in agricultural production (Supplementary Table 12). 1.0 $\mu \mathrm{L}$ drops of serial dilution of technical insecticides in acetone solution were applied with a micropipette to the thoracic dorsum of the 3rd instars and the control larvae were treated with $1.0 \mu \mathrm{L}$ acetone. After treatment, the larvae were reared individually in 24-well plates containing ad libitum artificial diet without any Bt proteins and insecticides. Larvae were retained in an insect chamber with a controlled environment of $26 \pm 1{ }^{\circ} \mathrm{C}, 60 \pm 10 \% \mathrm{RH}$ and a photoperiod of $16 \mathrm{~h}: 8 \mathrm{~h}$ (L: D). Mortality was assessed after $72 \mathrm{~h}$ treatment. Larvae were considered dead if they were unable to move in a coordinated manner when prodded with a small soft brush. We used median lethal doses $\mathrm{LC}_{50}$ to evaluate the resistance level of different fall armyworm populations. The $\mathrm{LC}_{50}$ for each assay of insecticides was estimated by probit analysis using the software package POLO-PC ${ }^{62}$ (LeOra Software, Berkeley, CA, USA).

The Bt toxin field bioassays to were conducted at a genetically modified (GM) test base in Yunnan Province, China. Test seeds of GM maize (expressing Cry1 Ab) and control maize were provided by DBN Biotech Center, Beijing DBN Technology Group Co., Ltd. Both maize types were planted in approximately $180 \mathrm{~m}^{2}$, with each type being replicated three times. Larval density and maize damage rates were investigated at different growth stages of maize at seven different dates during June to July. The investigation was performed in a five-spot-sampling method with 20 maize plants per point. Fall armyworm damage assessment was performed according to standard procedures $^{63-65}$.

\section{Acknowledgements}

The following bodies provided funding that contributed to this work: Key Project for Breeding Genetic Modified Organisms grant (2016ZX08012004-003), the UK's Global Challenges Research Fund and Biotechnology and Biological Sciences Research Council (BB/P023444/1), the UK Natural Environment Research Council Envision Doctoral Training Programme (NE/L002604/1). 
a
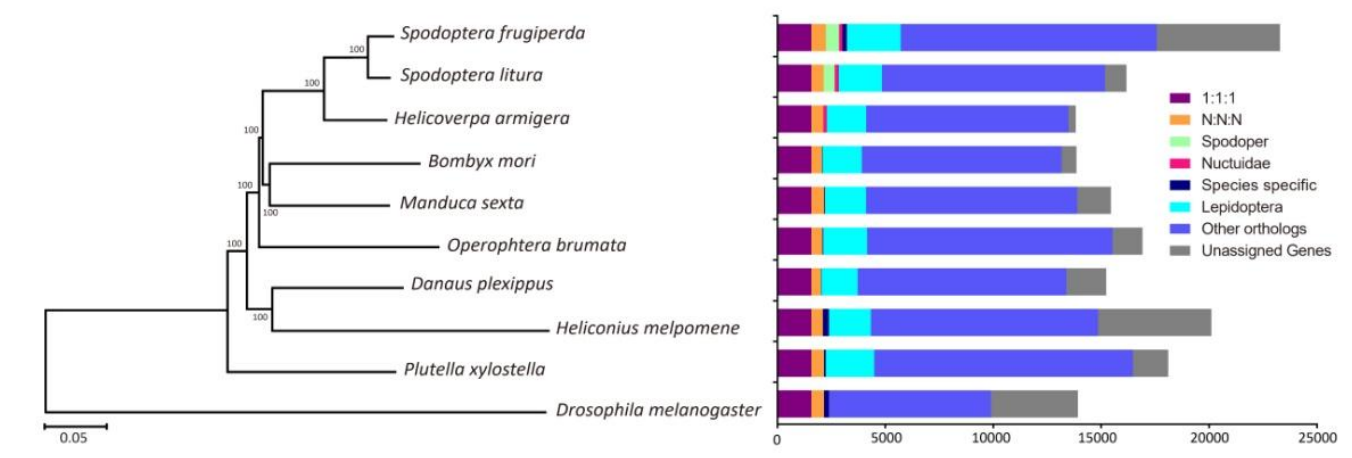

b

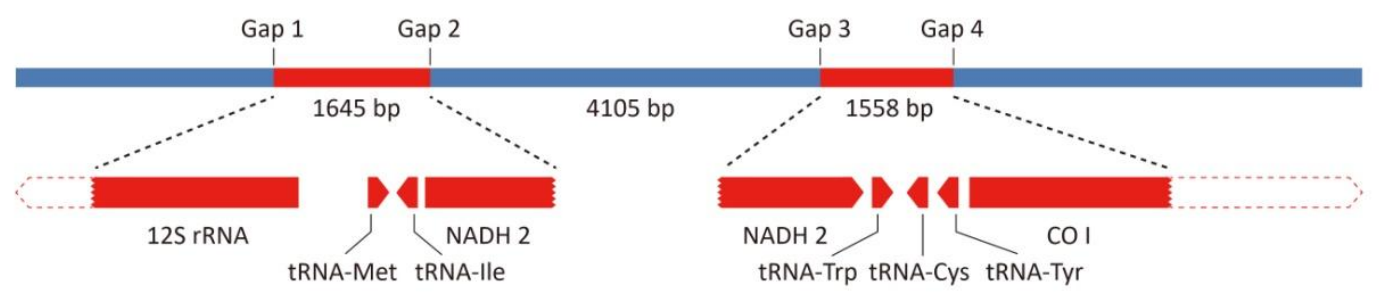

Figure 1. Phylogenetic relationships and schematic map of mitochondrial insertion. a) Phylogenetic tree and genomic comparison of 10 species of Lepidoptera and Diptera. Drosophila melanogaster was used as an outgroup and bootstrap value was set as 1000, 1:1:1 include the common orthologs with the same number of copies in different species, N:N:N include the common orthologs with different copy numbers in different species, other orthologs include the unclassified orthologs, and unassigned genes include the genes that cannot be clustered into known gene families. b) A schematic map of two mitochondrial fragments inserted into the nuclear genome, NADH2 gene was separated by a 4105-bp fragment, and both two inserted mitochondrial fragments were identical with C-strain genotype. 
bioRxiv preprint doi: https://doi org/101101/813154 t this version posted November 29, 2019. The copyright holder for this preprint (which was not certified by peer review) is the author/funder, who has granted bioRxiv a license to display the preprint in perpetuity. It is made available under aCC-BY-NC 4.0 International license.

a
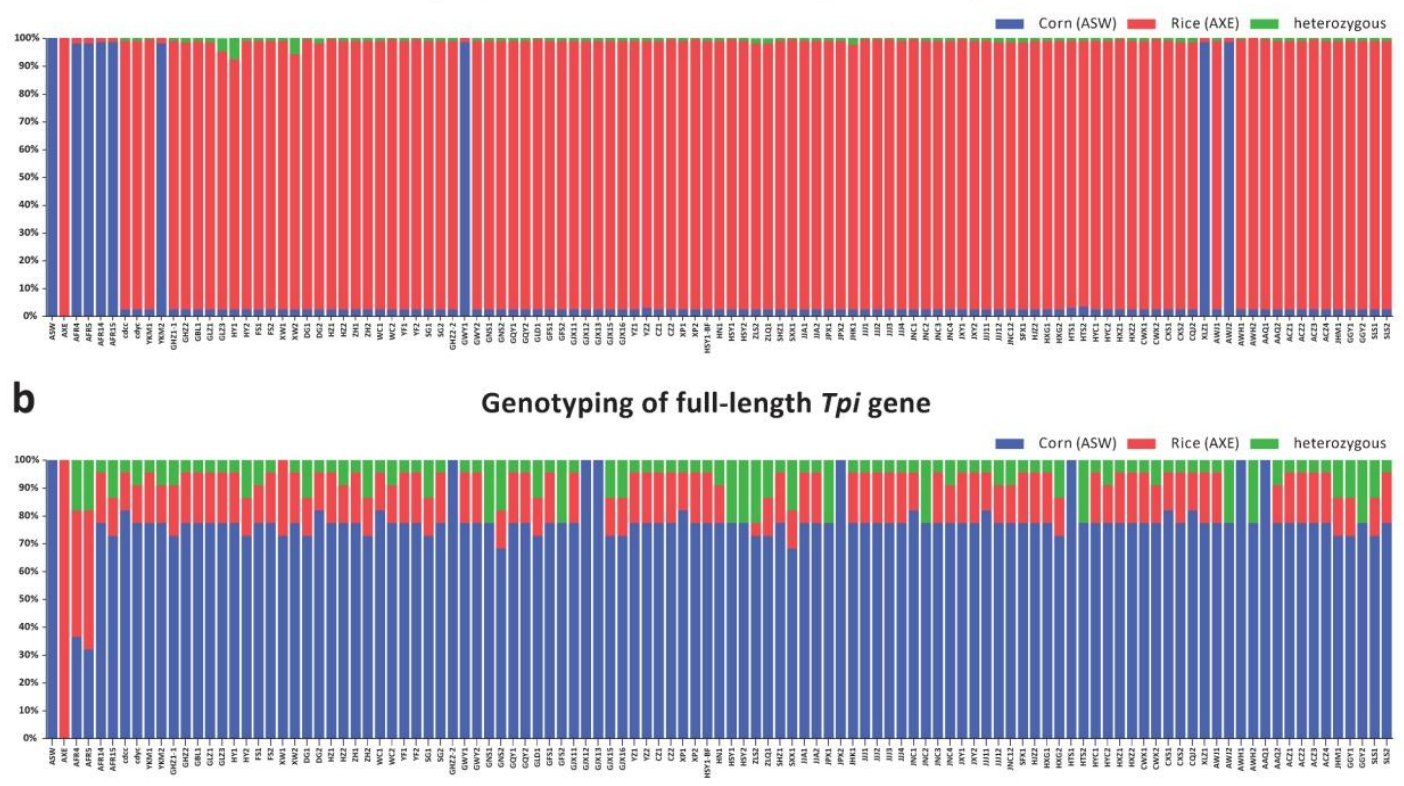

C Genotyping of whole genome SNPs

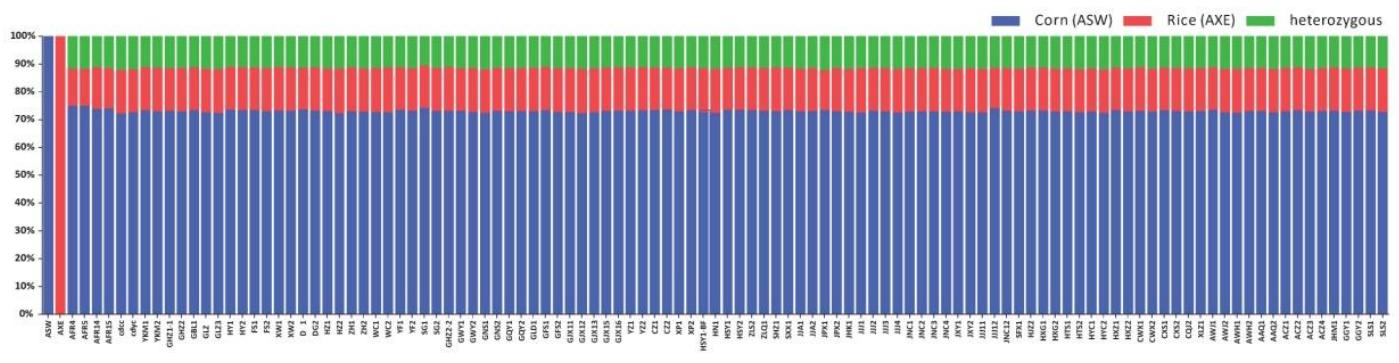

Figure 2. Genetic background of 109 fall armyworm samples based on two molecular markers and genome-wide SNPs. ASW represents the American corn strain and AXE represents the American rice strain. AFR4-5 were both from the inbred strain AFR2017 collected from Zambia, AFR14-15 were both from the same inbred strain AFR2019 collected from Malawi. cdcc and cdyc represent two inbred strains collected from Yunnan Province in China. 


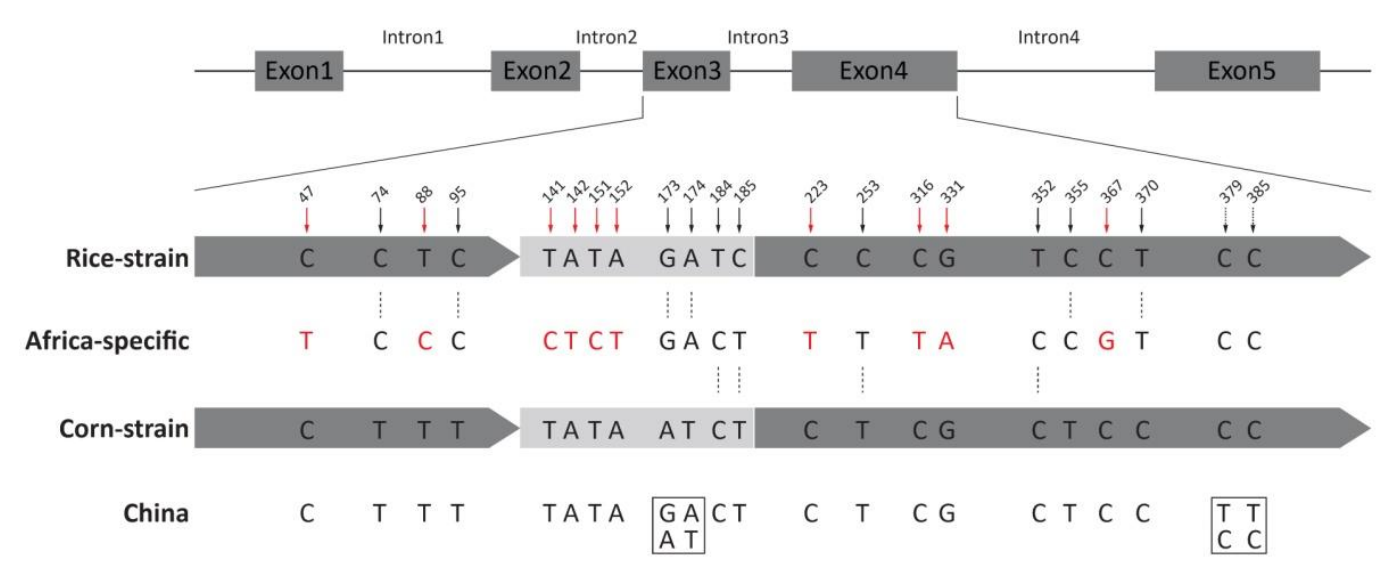

Figure 3. Diagram of the Tpi gene segments with respect to consensus Western Hemisphere sequences and the haplotypes observed in samples collected from Africa and China. Black solid arrows represent 10 SNPs used to identify traditional R-strain and C-strain fall armyworm, in which P370 was considered to be an effective diagnostic marker especially. Red solid arrows represent 10 SNPs specific to Africa-specific strain. The boxes represent two variable loci in some Chinese samples, including homozygous or heterozygous genotypes.

a

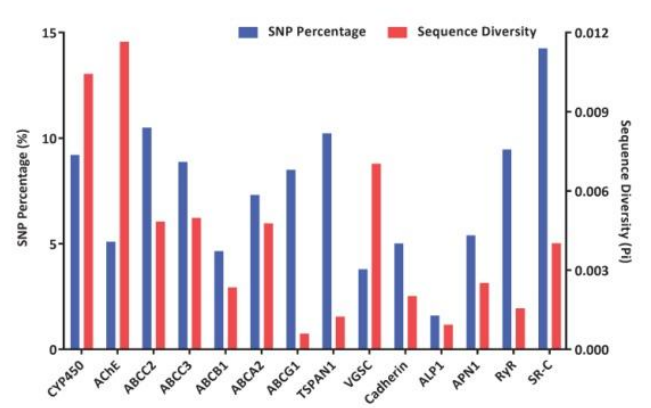

b

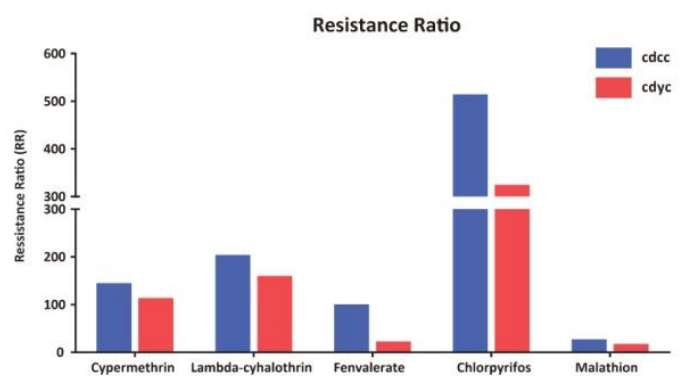

C
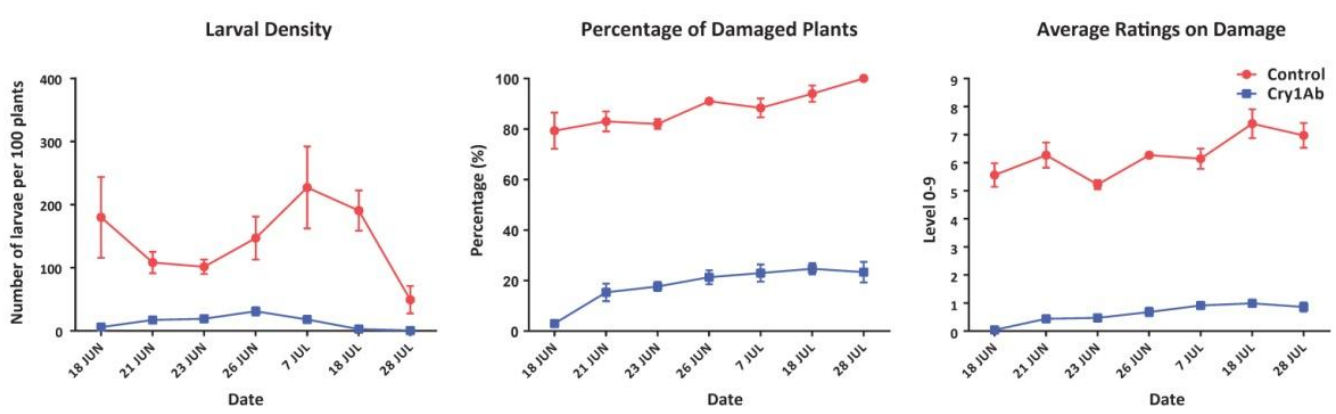

Figure 4. Genomic scanning and bioassays of fall armyworm for insecticides resistance. a) Sequence variation analysis of pesticides resistance and Bt resistance related genes in CDS region. SNP percentage means the proportion of variation sites in the complete gene CDS length. b) The resistance ratios (RRs) of two Chinese fall armyworm populations to pyrethroids (cypermethrin, lambda-cyhalothrin, fenvalerate) and organophosphates (chlorpyrifos, malathion) insecticides, The RRs was calculated by $\mathrm{LD}_{50}(\mu \mathrm{g} / \mathrm{g})$ of field population over the $\mathrm{LD}_{50}$ of the susceptible population. The $\mathrm{LD}_{50} \mathrm{~s}$ of the susceptible population here was referred to $\mathrm{Yu}$ et al. (1991). c) Resistance tests of GM maize and control maize to fall armyworm in field experiments. 
bioRxiv preprint doi: https://doi org/101101/813154: this version posted November 29,2019 . The copyright holder for this preprint (which was not certified by peer review) is the author/funder, who has granted bioRxiv a license to display the preprint in perpetuity. It is made available under aCC-BY-NC 4.0 International license.
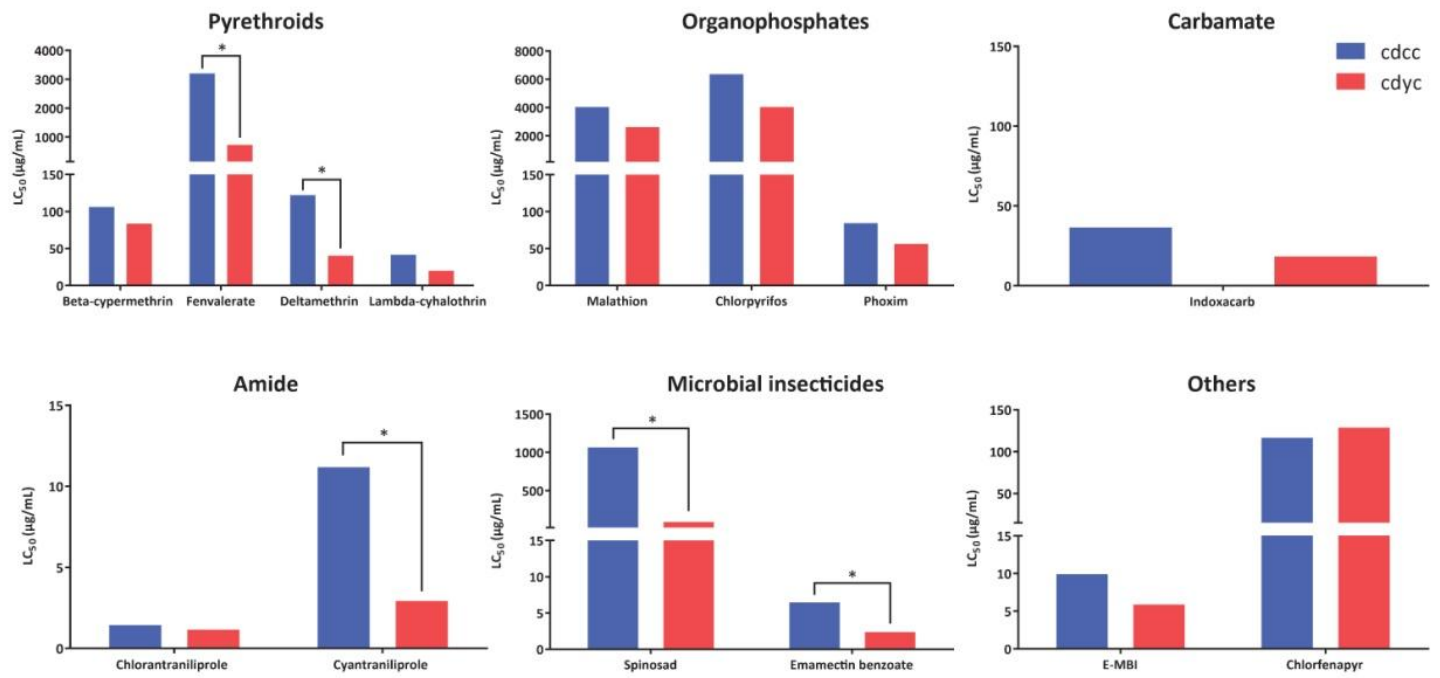

Figure 5. The $\mathrm{LC}_{50} \mathrm{~s}$ of two Chinese fall armyworm populations to different kinds of insecticides. The significantly difference was considered by whether the $95 \%$ FL have overlap $(* \mathrm{P}<0.05)$.

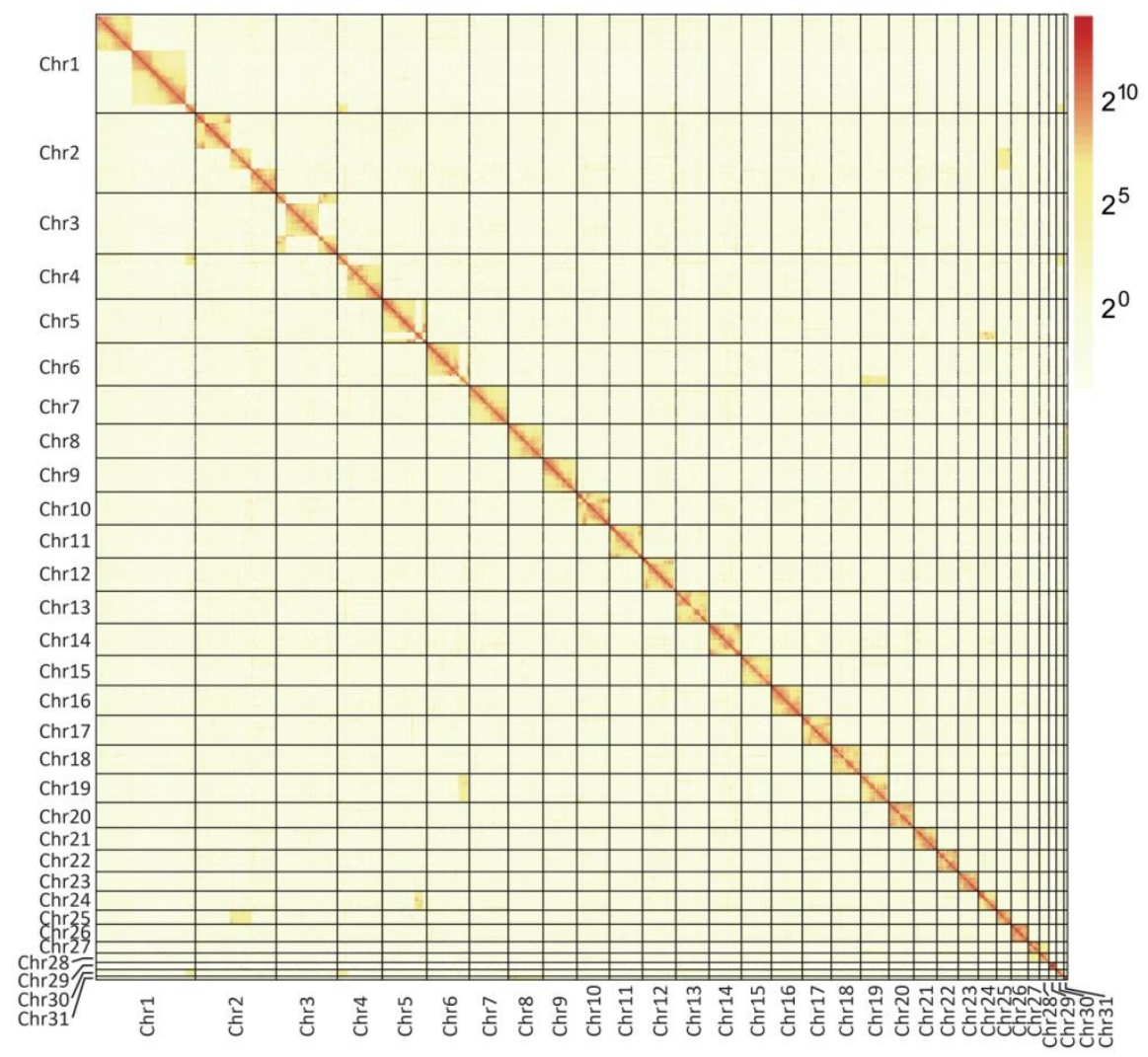

Supplementary Figure 1. A genome-wide contact matrix from Hi-C data between each pair of the 31 chromosomes. The color value indicates the number of valid reads. 


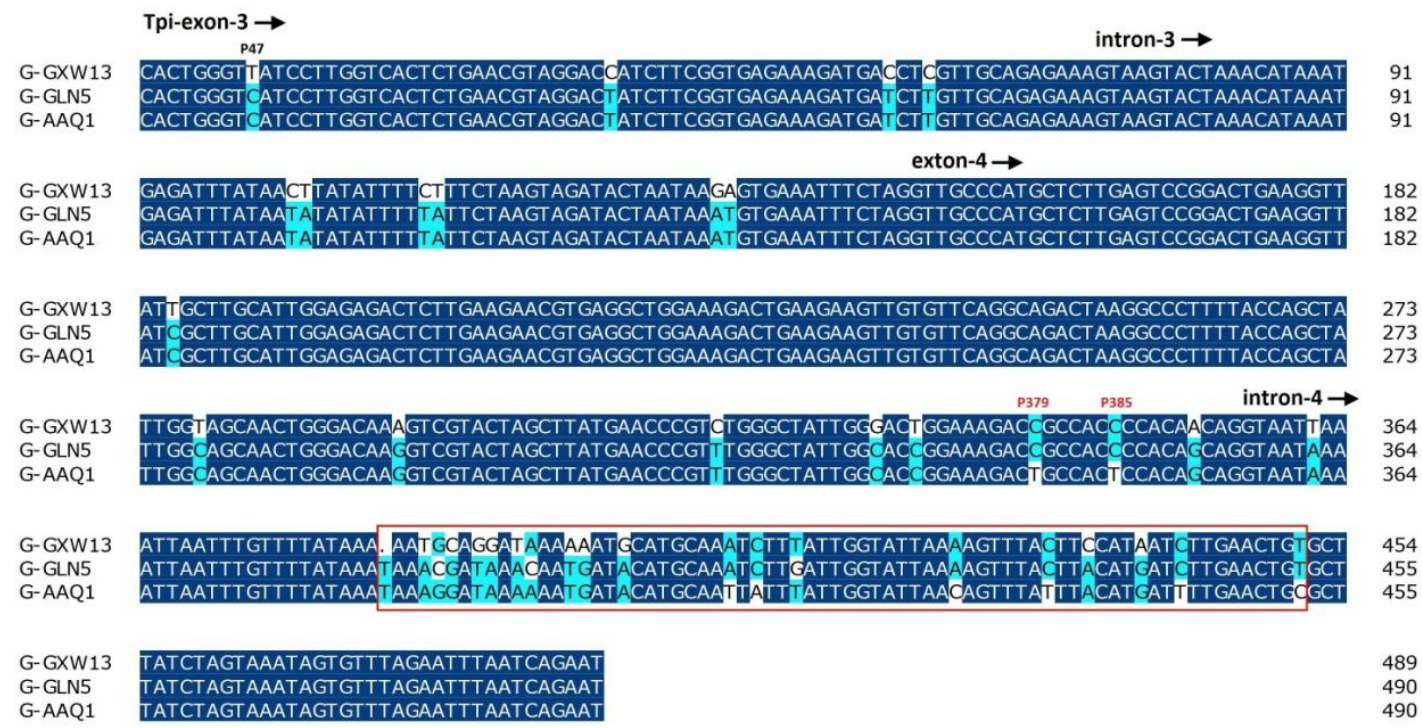

Supplementary Figure 2. Comparison of different Tpi genotypes in Chinese samples. G-GXW13 represents Africa-specific genotype, G-GLN5 and G-AAQ1 represent C-strain genotype with difference in intron4 region. Red box represents the sequence variation in intron4 region linked to two variable sites (P379/385) in exon-4 of Tpi gene.

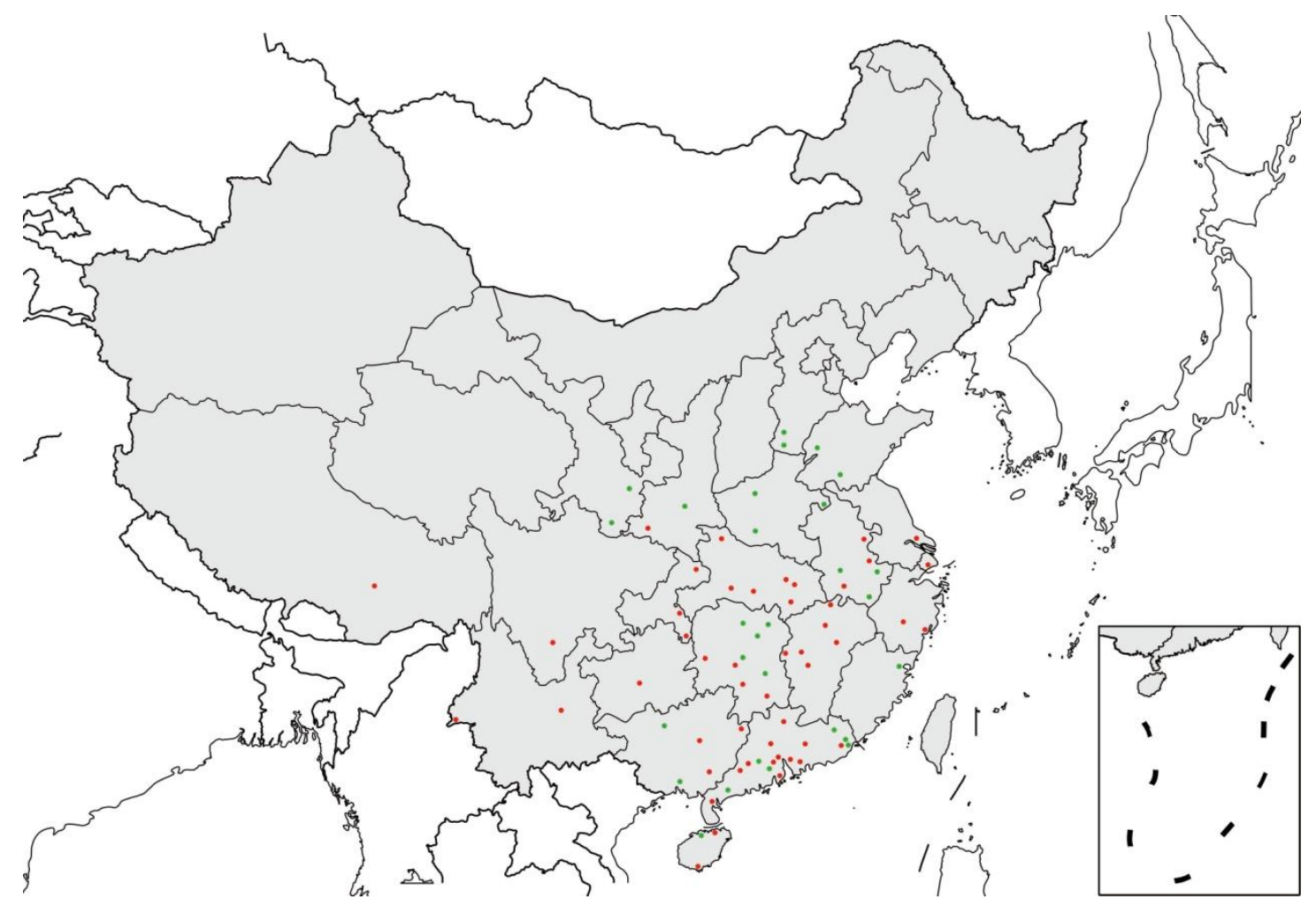

Supplementary Figure 3. Map of fall armyworm collection sites in China. Each point represents a city, the red point represents the source of the re-sequenced samples from 16 Provinces in China, the green points represents the source of some rest samples from other 5 Provinces used for population genetic study in this study. 
Table 1. Genotype and resistance mutation sites of pesticide-related genes in fall armyworm populations in China.

\begin{tabular}{|c|c|c|c|c|c|c|c|c|}
\hline & \multicolumn{8}{|c|}{ Genotype } \\
\hline Gene & \multicolumn{3}{|c|}{ AChE } & \multicolumn{3}{|c|}{ VGSC } & \multicolumn{2}{|c|}{ RyR } \\
\hline Mutation Sites & AA201 & AA227 & AA290 & AA929 & AA932 & AA1014 & AA4790 & AA4946 \\
\hline $\begin{array}{l}\text { Susceptible } \\
\text { (amino acid) }\end{array}$ & A & G & $\mathrm{F}$ & $\mathrm{T}$ & $\mathrm{L}$ & $\mathrm{L}$ & I & G \\
\hline $\begin{array}{l}\text { Resistance } \\
\text { (amino acid) }\end{array}$ & S & A & V & I & $\mathrm{F}$ & $\mathrm{F}$ & M & GE \\
\hline $\begin{array}{c}\text { Re-sequenced } \\
\text { Samples }\end{array}$ & $\begin{array}{c}\text { AA } \\
(82.9 \%) \\
\text { AS } \\
(17.1 \%)\end{array}$ & $\begin{array}{c}\text { GG } \\
(100 \%)\end{array}$ & $\begin{array}{c}\text { FF } \\
(12.1 \%) \\
\text { FV } \\
(58.2 \%) \\
\text { VV } \\
(29.7 \%)\end{array}$ & $\begin{array}{c}\text { TT } \\
(100 \%)\end{array}$ & $\begin{array}{c}\text { LL } \\
(100 \%)\end{array}$ & $\begin{array}{c}\text { LL } \\
(100 \%)\end{array}$ & $\begin{array}{c}\text { II } \\
(100 \%)\end{array}$ & $\begin{array}{c}\text { GG } \\
(100 \%)\end{array}$ \\
\hline
\end{tabular}

Supplementary Table 1. Statistical results of chromosome lengths by Hi-C assembly.

Supplementary Table 2. Comparison between two versions of published fall armyworm genomes and the genome of this study.

Supplementary Table 3. Annotation and distribution of repetitive elements in fall armyworm genome.

Supplementary Table 4. Summary of assembly and annotation of Lepidoptera and Diptera genomes.

Supplementary Table 5. Information of $\mathbf{1 7 3}$ fall armyworm samples used for population genetic analysis in this study.

Supplementary Table 6. Distribution of mitochondrial insertion in 109 re-sequenced fall armyworm samples and American corn-strain (ASW) and rice-strain (AXE).

Supplementary Table 7. Genes related to pesticide resistance and Bt resistance in fall armyworm.

Supplementary Table 8. The $\mathrm{LC}_{50} \mathrm{~s}$ of two Chinese fall armyworm populations to 14 kinds of insecticides.

Supplementary Table 9. The resistance ratios of two Chinese fall armyworm populations to five kinds of pyrethroids and organophosphorus insecticides. The resistance ratio was calculated by $\mathrm{LD}_{50}(\mu \mathrm{g} / \mathrm{g})$ of field population over the $\mathrm{LD}_{50}$ of the susceptible population.

Supplementary Table 10. Information of 109 re-sequenced fall armyworm samples in this study.

Supplementary Table 11. Primer sequence information used in this study.

Supplementary Table 12. General information of $\mathbf{1 4}$ kinds of insecticides used in topical bioassays. 


\section{References}

1 Nagoshi, R. N., Meagher, R. L. \& Hay-Roe, M. Inferring the annual migration patterns of fall armyworm (Lepidoptera: Noctuidae) in the United States from mitochondrial haplotypes. Ecology and evolution 2, 1458-1467 (2012).

2 Mitchell, E. R. et al. Seasonal periodicity of fall armyworm, (Lepidoptera: Noctuidae) in the Caribbean basin and northward to Canada. Journal of entomological science 26, 39-50 (1991).

3 Johnson, S. J. Migration and the life history strategy of the fall armyworm, Spodoptera frugiperda in the Western Hemisphere. International Journal of Tropical Insect Science $\mathbf{8}$, 543-549 (1987).

4 Westbrook, J. K., Nagoshi, R. N., Meagher, R. L., Fleischer, S. J. \& Jairam, S. Modeling seasonal migration of fall armyworm moths. International journal of biometeorology $\mathbf{6 0}$, 255-267 (2016).

5 Goergen, G., Kumar, P. L., Sankung, S. B., Togola, A. \& Tamo, M. First report of outbreaks of the fall armyworm Spodoptera frugiperda (J. E. Smith) (Lepidoptera, Noctuidae), a new alien invasive pest in West and Central Africa. PloS one 11, e0165632 (2016).

6 Luginbill, P. The fall armyworm. U.S. Dept. Agric. Tech. Bull. 34, 1-91 (1928).

7 Pitre, H. N. Relationship of fall armyworm (Lepidoptera: Noctuidae) from Florida, Honduras, Jamaica, and Mississippi: susceptibility to insecticides with reference to migration. Florida Entomologist 71, 56-61 (1988).

8 Leibee, G. L. \& Capinera, J. L. Pesticide resistance in Florida insects limits management options. Florida Entomologist 78, 386-399 (1995).

9 Burtet, L. M. et al. Managing fall armyworm, Spodoptera frugiperda (Lepidoptera: Noctuidae), with Bt maize and insecticides in southern Brazil. Pest management science 73, 2569-2577 (2017).

10 Blanco, C. A. et al. Susceptibility of isofamilies of Spodoptera frugiperda (Lepidoptera: Noctuidae) to Cry1Ac and Cry1Fa proteins of Bacillus thuringiensis. Southwestern Entomologist 35, 409-416 (2010).

11 Storer, N. P. et al. Discovery and characterization of field resistance to Bt maize: Spodoptera frugiperda (Lepidoptera: Noctuidae) in Puerto Rico. Journal of economic entomology 103, 1031-1038 (2010).

12 Monnerat, R. et al. Evidence of field-evolved resistance of Spodoptera frugiperda to Bt corn expressing Cry1F in Brazil that is still sensitive to modified Bt toxins. PLoS one 10, e0119544 (2015).

13 Fatoretto, J. C., Michel, A. P., Silva Filho, M. C. \& Silva, N. Adaptive potential of fall armyworm (Lepidoptera: Noctuidae) limits Bt trait durability in Brazil. Journal of Integrated Pest Management 8, 1-10 (2017).

14 Signorini, A. M. et al. Management of field-evolved resistance to Bt maize in Argentina: a multi-institutional approach. Frontiers in bioengineering and biotechnology 6, 1-5 (2018).

15 Chen, X. et al. Fitness costs of Vip3A resistance in Spodoptera frugiperda on different hosts. Pest management science 75, 1074-1080 (2019).

16 Bernardi, O. et al. Frequency of resistance to Vip3Aa20 toxin from Bacillus thuringiensis in Spodoptera frugiperda (Lepidoptera: Noctuidae) populations in Brazil. Crop Protection 76, 7-14 (2015). 
17 Gouin, A. et al. Two genomes of highly polyphagous lepidopteran pests (Spodoptera frugiperda, Noctuidae) with different host-plant ranges. Scientific reports 7, 1-12 (2017).

$18 \mathrm{Liu}, \mathrm{H}$. et al. et al. Chromosome level draft genomes of the fall armyworm, Spodoptera frugiperda (Lepidoptera: Noctuidae), an alien invasive pest in China. bioRxiv doi: https://doi.org/10.1101/671560 (2019).

19 Pashley, D. P. \& Martin, J. A. Reproductive incompatibility between host strains of the fall armyworm (Lepidoptera: Noctuidae). Annals of the Entomological Society of America 80, 731-733 (1987).

20 Pashley, D. P. Host-associated genetic differentiation in fall armyworm (Lepidoptera: Noctuidae): a sibling species complex? Annals of the Entomological Society of America 79, 898-904 (1986).

21 Lima, E. R. \& Mcneil, J, N. Female sex pheromones in the host races and hybrids of the fall armyworm, Spodoptera frugiperda (Lepidoptera: Noctuidae). Chemoecology 19, 29-36 (2009).

22 Pashley, D. P., Hammond, A. M. \& Hardy, T. N. Reproductive isolating mechanisms in fall armyworm host strains (Lepidoptera, Noctuidae). Annals of the Entomological Society of America 85, 400-405 (1992).

23 Nagoshi, R. N., Meagher, R. L., Nuessly, G. \& Hall, D. G. Effects of fall armyworm (Lepidoptera: Noctuidae) interstrain mating in wild populations. Environmental entomology $\mathbf{3 5}$, 561-568 (2006).

24 Dumas, P. et al. Spodoptera frugiperda (Lepidoptera: Noctuidae) host-plant variants: two host strains or two distinct species? Genetica 143, 305-316 (2015).

$25 \mathrm{Zhu}$, Y. C. et al. Evidence of multiple/cross resistance to Bt and organophosphate insecticides in Puerto Rico population of the fall armyworm, Spodoptera frugiperda. Pesticide Biochemistry and Physiology 122, 15-21 (2015).

26 Huang, F. et al. Frequency of Bacillus thuringiensis Cry1A. 105 resistance alleles in field populations of the fall armyworm, Spodoptera frugiperda, in Louisiana and Florida. Crop Protection 83, 83-89 (2016).

$27 \mathrm{Li}, \mathrm{G}$. et al. Frequency of Cry1F non-recessive resistance alleles in North Carolina field populations of Spodoptera frugiperda (Lepidoptera: Noctuidae). PloS one 11, e0154492 (2016).

28 Chandrasena, D. I. et al. Characterization of field-evolved resistance to Bacillus thuringiensis-derived Cry1F $\delta$ - endotoxin in Spodoptera frugiperda populations from Argentina. Pest management science 74, 746-754 (2018).

29 Gutiérrez-Moreno, R. et al. Field-evolved resistance of the fall armyworm (Lepidoptera: Noctuidae) to synthetic insecticides in Puerto Rico and Mexico. Journal of economic entomology 112, 792-802 (2019).

30 Yu, S. J., Nguyen, S. N. \& Abo-Elghar, G. E. Biochemical characteristics of insecticide resistance in the fall armyworm, Spodoptera frugiperda (J. E. Smith). Pesticide Biochemistry and Physiology 77, 1-11 (2003).

31 Carvalho, R. A., Omoto, C., Field, L. M., Williamson, M. S. \& Bass, C. Investigating the molecular mechanisms of organophosphate and pyrethroid resistance in the fall armyworm Spodoptera frugiperda. PLoS One 8, e62268 (2013).

32 Boaventura, D. et al. Detection of a ryanodine receptor target-site mutation in diamide 
insecticide resistant fall armyworm, Spodoptera frugiperda. Pest management science, 1-8 (2019).

33 Banerjee, R. et al. Mechanism and DNA-based detection of field-evolved resistance to transgenic Bt corn in fall armyworm (Spodoptera frugiperda). Scientific reports 7, 1-10 (2017).

34 Yang, F. et al. F2 screen, inheritance and cross-resistance of field-derived Vip3A resistance in Spodoptera frugiperda (Lepidoptera: Noctuidae) collected from Louisiana, USA. Pest management science 74, 1769-1778 (2018).

35 Meagher, R. L. \& Gallo-Meagher, M. Identifying host strains of fall armyworm (Lepidoptera: Noctuidae) in Florida using mitochondrial markers. Florida Entomologist 86, 450-455 (2003).

36 Nagoshi, R. N. \& Meagher, R. L. Using intron sequence comparisons in the triose-phosphate isomerase gene to study the divergence of the fall armyworm host strains. Insect molecular biology 25, 324-337 (2016).

37 Nagoshi, R. N. Improvements in the identification of strains facilitate population studies of fall armyworm subgroups. Annals of the Entomological Society of America 105, 351-358 (2012).

38 Nagoshi, R. N. et al. Comparative molecular analyses of invasive fall armyworm in Togo reveal strong similarities to populations from the eastern United States and the Greater Antilles. PLoS One 12, e0181982 (2017).

39 Jua'ren, M. L. et al. Population structure of Spodoptera frugiperda maize and rice host forms in South America: are they host strains? Entomologia Experimentalis et Applicata 152, 182-199 (2014)

40 Nagoshi, R. N., Goergen, G., Du Plessis, H., van den Berg, J. \& Meagher, R., Jr. Genetic comparisons of fall armyworm populations from 11 countries spanning sub-Saharan Africa provide insights into strain composition and migratory behaviors. Scientific reports $\mathbf{9}, 1-11$ (2019).

41 Nagoshi, R. N. Evidence that a major subpopulation of fall armyworm found in the Western Hemisphere is rare or absent in Africa, which may limit the range of crops at risk of infestation. PloS one 14, e0208966 (2019).

42 Zhang, L. et al. Molecular characterization analysis of fall armyworm populations in China. Plant Protection 45, 10-27 (2019).

43 Kakumani, P. K., Malhotra, P., Mukherjee, S. K. \& Bhatnagar, R. K. A draft genome assembly of the army worm, Spodoptera frugiperda. Genomics 104, 134-143 (2014).

44 Nandakumar, S., Ma, H. \& Khan, A. S. Whole-genome sequence of the Spodoptera frugiperda Sf9 insect cell line. Genome Announcements 5, e00829-17 (2017).

45 Ruan, J. \& Li, H. Fast and accurate long-read assembly with wtdbg2. bioRxiv doi: https://doi.org/10.1101/530972 (2019)

46 Zhan, S., Merlin, C., Boore, J. L. \& Reppert, S. M. The monarch butterfly genome yields insights into long-distance migration. Cell 147, 1171-1185 (2011).

47 Dasmahapatra, K. K. et al. Butterfly genome reveals promiscuous exchange of mimicry adaptations among species. Nature 487, 94-98 (2012).

48 The International Silkworm Genome Consortium. The genome of a lepidopteran model insect, the silkworm Bombyx mori. Insect biochemistry and molecular biology 38, 1036-1045 (2008).

49 Pearce, S. L. et al. Genomic innovations, transcriptional plasticity and gene loss underlying the evolution and divergence of two highly polyphagous and invasive Helicoverpa pest 
species. BMC biology 15, 1-30 (2017).

50 You, M. et al. A heterozygous moth genome provides insights into herbivory and detoxification. Nature genetics 45, 220-227 (2013).

51 Kanost, M. R. et al. Multifaceted biological insights from a draft genome sequence of the tobacco hornworm moth, Manduca sexta. Insect biochemistry and molecular biology 76, 118-147 (2016).

$52 \mathrm{Yu}$, S. J. Insecticide resistance in the fall armyworm, Spodoptera frugiperda (J. E. Smith). Pesticide Biochemistry and Physiology 39, 84-91 (1991).

$53 \mathrm{Li}$, G. P. et al. Susceptibility evaluation of invaded Spodoptera frugiperda population in Yunnan province to five Bt proteins. Plant Protection 45, 15-20 (2019).

54 Burton, J. N. et al. Chromosome-scale scaffolding of de novo genome assemblies based on chromatin interactions. Nature biotechnology 31, 1119-1127 (2013).

55 Benson, G. Tandem repeats finder: a program to analyze DNA sequences. Nucleic acids research 27, 573-580 (1999).

56 Haas, B. J. et al. Automated eukaryotic gene structure annotation using EVidenceModeler and the Program to Assemble Spliced Alignments. Genome biology 9, 1-22 (2008).

57 Edgar, R. C. MUSCLE: multiple sequence alignment with high accuracy and high throughput. Nucleic acids research 32, 1792-1797 (2004).

$58 \mathrm{Li}$, H. \& Durbin, R. Fast and accurate short read alignment with Burrows-Wheeler transform. bioinformatics 25, 1754-1760 (2009).

$59 \mathrm{Li}$, H. et al. The sequence alignment/map format and SAMtools. Bioinformatics 25, 2078-2079 (2009).

$60 \mathrm{Li}, \mathrm{H}$. A statistical framework for SNP calling, mutation discovery, association mapping and population genetical parameter estimation from sequencing data. Bioinformatics 27, 2987-2993 (2011).

61 Armes, N. J., Jadhav, D. R., Bond, G. S. \& King, A. B. Insecticide resistance in Helicoverpa armigera in South India. Pesticide science 34, 355-364 (1992).

62 Russell, R. M., Robertson, J. L. \& Savin, N. E. POLO: a new computer program for probit analysis. Bulletin of the ESA 23, 209-213 (1977).

63 Davis, F. M., Ng, S. S. \& Williams, W. P. Visual rating scales for screening whorl-stage corn for resistance to fall armyworm. Technical bulletin-Mississippi Agricultural and Forestry Experiment Station (USA) (1992).

64 Williams, W. P., Buckley, P. M. \& Daves, C. A. Identifying resistance in corn to southwestern corn borer (Lepidoptera: Crambidae), fall armyworm (Lepidoptera: Noctuidae), and corn earworm (Lepidoptera: Noctuidae). J. Agr. Urban Entomol 23, 87-95 (2006).

65 Wiseman, B. R. \& Widstrom, N. W. Fall armyworm damage ratings on corn at various infestation levels and plant development stages. J. Agric. Entomol 1, 115-119 (1984). 